Research

\title{
Metformin improves hepatic IRS2/PI3K/Akt signaling in insulin-resistant rats of NASH and cirrhosis
}

\author{
Hong Xu1, Yang Zhou'2,3, Yongxia Liư ${ }^{4}$, Jian Ping'2,3, Qiyang Shou ${ }^{5}$, Fangming Chen ${ }^{5}$ \\ and Ru Ruo 6 \\ 1Department of Gastroenterology and Hepatology, Hangzhou Red Cross Hospital, Hangzhou, China \\ 2Liver Cirrhosis Section, Department of Hepatology, Shuguang Hospital Affiliated to Shanghai \\ University of Traditional Chinese Medicine, Shanghai, China \\ ${ }^{3}$ Institute of Liver Diseases, Shanghai University of Traditional Chinese Medicine, Shanghai, China \\ ${ }^{4}$ Department of Clinical Laboratory, Hangzhou Red Cross Hospital, Hangzhou, China \\ ${ }^{5}$ Experimental Animal Research Center, Zhejiang Chinese Medical University, Hangzhou, China \\ ${ }^{6}$ Department of Pathology, Hangzhou Red Cross Hospital, Hangzhou, China
}

Correspondence should be addressed to $\mathrm{HXu}$ Email hongxuhzrc@aliyun.com

\begin{abstract}
Nonalcoholic fatty liver disease and cirrhosis are strongly associated with insulin resistance and glucose intolerance. To date, the influence of metformin on glycogen synthesis in the liver is controversial. Limited studies have evaluated the effect of metformin on hepatic insulin signaling pathway in vivo. In this study, an insulin-resistant rat model of nonalcoholic steatohepatitis and cirrhosis was developed by high-fat and high-sucrose diet feeding in combination with subcutaneous injection of carbon tetrachloride. Liver tissues of the model rats were featured with severe steatosis and cirrhosis, accompanied by impaired liver function and antioxidant capacity. The glucose tolerance was impaired, and the index of insulin resistance was increased significantly compared with the control. The content of hepatic glycogen was dramatically decreased. The expression of insulin receptor $\beta$ (IR $\beta$ ); phosphorylations of IR $\beta$, insulin receptor substrate 2 (IRS2), and Akt; and activities of phosphatidylinositol 3-kinase (PI3K) and glycogen synthase (GS) in the liver were significantly decreased, whereas the activities of glycogen synthase kinase $3 \alpha(\mathrm{GSK} 3 \alpha)$ and glycogen phosphorylase a (GPa) were increased. Metformin treatment remarkably improved liver function, alleviated lipid peroxidation and histological damages of the liver, and ameliorated glucose intolerance and insulin resistance. Metfromin also significantly upregulated the expression of IR $\beta$; increased the phosphorylations of IR $\beta$, IRS2, and Akt; increased the activities of PI3K and GS; and decreased GSK $3 \alpha$ and GPa activities. In conclusion, our study suggests that metformin upregulates IR $\beta$ expression and the downstream IRS2/PI3K/Akt signaling transduction, therefore, to increase hepatic glycogen storage and improve insulin resistance. These actions may be attributed to the improved liver histological alterations by metformin.
\end{abstract}

Key Words

- metformin

- insulin resistance

- IRS2/PI3K/Akt signaling pathway

- cirrhosis

- nonalcoholic steatohepatitis
Published by Bioscientifica Ltd
Journal of Endocrinology

(2016) 229, 133-144 


\section{Introduction}

The liver is a primary target organ for insulin and plays a critical role in glucose homeostasis. It maintains a balance between the uptake and storage of glucose by regulating glycogenesis and the production of glucose by glycogenolysis and gluconeogenesis (Radziuk \& Pye 2001). Nonalcoholic fatty liver disease (NAFLD) is a spectrum of diseases ranging from simple steatosis to nonalcoholic steatohepatitis (NASH), which may progress to cirrhosis. NAFLD is strongly associated with insulin resistance, and up to $50 \%$ of NAFLD patients have type 2 diabetes (Dyson \& Day 2014). A significant proportion of patients with cirrhosis ranging between 35 and 80\% may be insulin resistant, and $20-30 \%$ of patients may be clinically diabetic (Ahmadieh \& Azar 2014).

In the liver, the insulin receptor substrate 2 (IRS2)/phosphatidylinositol 3-kinase (PI3K)/protein kinase B (Akt) signaling transduction pathway plays a pivotal role in modulating the glucose metabolic actions of insulin (Pessin \& Saltiel 2000). Insulin receptor (IR) is a membrane protein composed of two extracellular $\alpha$-subunits and two transmembrane $\beta$-subunits, which contain intracellular tyrosine kinase domains. Binding of insulin to the $\alpha$-subunit increases the intrinsic tyrosine kinase activity and initiates autophosphorylation of the $\beta$-subunit, triggering a cascade of phosphorylation and activation of essential downstream targets, including IRS2, PI3K, and Akt (Valverde et al. 2003, Ide et al. 2004). The activation of Akt causes the phosphorylation and inactivation of glycogen synthase kinase $3 \alpha$ (GSK3 $\alpha$ ). GSK3 is the first identified Akt substrate and refers to two isoforms, GSK3 $\alpha$ and GSK3 $\beta$. GSK3 $\alpha$ is mainly involved in the process of glycogen synthesis, whereas GSK3 $\beta$ exhibits more complex biological functions (Beurel et al. 2015). The inactivation of GSK3 $\alpha$ induces the activation of glycogen synthase (GS). PI3K/Akt activation also leads to the conversion of glycogen phosphorylase (GP) from the active state $(\mathrm{GPa})$ to the inactive state $(\mathrm{GPb})$. The activation of GS and the depletion of GPa finally result in the stimulation of glycogen synthesis (Agius 2015).

Metformin, a potent biguanide hypoglycemic agent, has been clinically used for over five decades and is considered the first-line oral therapy for type 2 diabetes. Plenty of studies demonstrate that metformin improves glucose metabolism by suppressing hepatic glucose production mainly through inhibition of gluconeogenesis (Viollet et al. 2012). A number of potential mechanisms of decreasing hepatic gluconeogenesis by metformin have been proposed, including inhibition of mitochondrial respiratory chain activity, activation of AMP-activated protein kinase (AMPK), decrease in hepatic energy state, and inhibition of cAMP signaling through phosphorylation of CREB-binding protein (He et al. 2009, Cao et al. 2014, Madiraju et al. 2014). However, the precise molecular targets of metformin action still remain elusive. Few studies have examined the effect of metformin on hepatic glycogen synthesis, and the results are controversial. Increased hepatic glycogen content in response to metformin was detected in high-fat-fed, insulin-resistant rat model and in hepatoma cells. Also it has been reported that metformin impaired glycogen synthesis in isolated rat hepatocytes (Purrello et al. 1988, Mithieux et al. 2002, Otto et al. 2003). No in vivo data on the influence of metformin on IRS2/PI3K/Akt signaling pathway is available.

In this study, an insulin resistance-associated rat model of NASH and cirrhosis was developed and treated with metformin. The glycogen content in the liver was determined. The expression, phosphorylation status, and the activity of the key functional molecules in IRS2/PI3K/ Akt signaling pathway were detected. Our aim was to evaluate the influence of metformin on hepatic glycogen storage and insulin signaling transduction pathway.

\section{Materials and methods}

\section{Animals and chemicals}

Male Spraque-Dawley (SD) rats were obtained from SinoBritish SIPPR/BK Lab Animal Ltd, Shanghai, China (license no. SCXK 2008-0016) and reared at the Experimental Animal Research Center, Zhejiang Chinese Medical University, Hangzhou, China. Animals were maintained in the specific pathogen-free facility in accordance with the Institutional Animal Care Guidelines. The protocol was reviewed and approved by the Committee on Animal Research and Ethics of Zhejiang Chinese Medical University (authorization number: ZSLL-2012-058). All efforts were made to minimize suffering. Animals were killed under sodium pentobarbital anesthesia in a separate room and terminally bled via ventral aorta puncture.

Carbon tetrachloride $\left(\mathrm{CCl}_{4}\right)$ (289116) was purchased from Sigma-Aldrich. Metformin was purchased from SinoAmerican Shanghai Squibb Pharmaceuticals (Shanghai, China). Enzyme-linked immunosorbent assay (ELISA) kit (50R-E.1637) for determining insulin levels was obtained from Biovalue (Shanghai, China). Activity assay kits for PI3K (GMS50058.2), GSK3 $\alpha$ (GMS50161.2),

Published by Bioscientifica Ltd. 
GS (GMS50500.2), and GPa (GMS50092.4) and glycogen assay kit (GMS70085.2) were purchased from Genmed Scientifics (Shanghai, China). Malondialdehyde (MDA; A003-1), superoxide dismutase (SOD; A001-2), reduced glutathione (GSH; A006-1), and glutathione peroxidase (GSH-Px; A005) assay kits were purchased from Nanjing Jiancheng Biotechnology Institute, Nanjing, China. Bicinchoninic acid (BCA) protein assay kit (23227) was purchased from Pierce. Mouse anti-IR $\beta$ monoclonal antibody (ab69508) was obtained from Abcam. Rabbit anti-IRS2 (3089), rabbit anti-phospho Akt (Ser ${ }^{473}$ ) (4060), and rabbit anti-IR $\beta$ monoclonal antibody (3025) were purchased from Cell Signaling Technology. Mouse antiphosphotyrosine (PY) monoclonal antibody (05-321) was obtained from Millipore. Rabbit anti-Akt (sc-8312) and Protein A/G plus-agarose beads (sc-2003) were obtained from Santa Cruz Biotechnology.

\section{Developing insulin resistance-associated rat model of NASH and cirrhosis}

Thirty male SD rats weighing 180-200 g were equally randomized into three groups: control group $(n=10)$, model group $(n=10)$, and metformin-treated group $(n=10)$. Cirrhosis was induced by high-fat and high-sucrose diet (HFHSD) feeding in combination with subcutaneous injection of $\mathrm{CCl}_{4}$. The rats in the model group and metformin-treated group were fed with HFHSD, which consist of $74.25 \%$ standard chow, 10\% sucrose, $0.5 \%$ cholesterol, 5\% egg yolk powder, $10 \%$ lard, and $0.25 \%$ sodium cholate. Meanwhile, these rats received a single injection of $40 \% \mathrm{CCl}_{4}$ dissolved in olive oil at $5 \mathrm{~mL} / \mathrm{kg}$ and then $3 \mathrm{~mL} / \mathrm{kg}$ twice every week for 12 weeks. After 4 weeks of $\mathrm{CCl}_{4}$ exposure, the rats in the metformin-treated group additionally received intragastric administration of metformin twice a day at a dose of $200 \mathrm{mg} / \mathrm{kg} /$ day. The control rats were fed a standard chow, and received olive oil subcutaneously and normal saline intragastrically. The standard laboratory chow contains $20.5 \%$ protein, $4.62 \%$ fat, $52.5 \%$ nitrogen-free extract, and $4.35 \%$ fiber. Food and water were available ad libitum throughout the study.

\section{Measurement of serum biochemical parameters}

Blood samples without anticoagulant from the inferior vena cava were centrifuged at $2000 \boldsymbol{g}$ for $10 \mathrm{~min}$ to collect the serum. The activities of serum alanine aminotransferase (ALT), aspartate aminotransferase (AST), and alkaline phosphatase (ALP), and the levels of serum albumin (Alb), total bilirubin (TBil), and total cholesterol
(TC) were detected using an Olympus AU5400 automatic biochemical analyzer.

\section{Oxidative stress biomarker and antioxidant profile}

Liver samples were homogenized in $0.1 \mathrm{M}$ ice-cold phosphate buffer saline ( $\mathrm{pH} 7.4$ ). The homogenates were centrifuged at $14,000 \mathrm{~g}$ for $10 \mathrm{~min}$ at $4^{\circ} \mathrm{C}$. The supernatant was used for measuring MDA, SOD, GSH, and GSH-Px using commercial kits according to the manufacturer's protocols.

\section{Hematoxylin and eosin, Sirius red, and periodic acid-Schiff staining}

Liver specimens were fixed in $4 \%$ paraformaldehyde and dehydrated in a graded alcohol series. After embedded in paraffin, the tissues were sectioned at $4 \mu \mathrm{m}$ in thickness and stained with hematoxylin and eosin (H\&E). For evaluation of hepatic collagen deposition, Sirius red staining was performed and a red color staining was considered where the collagen deposited. Glycogen storage was detected by periodic acid-Schiff (PAS) staining staining as described elsewhere (Saitoh et al. 2010).

\section{Immunohistochemistry assessment}

After deparaffinization and rehydration, microwave antigen retrieval was performed on the sections as per a standard protocol before peroxidase quenching with 3\% hydrogen peroxide. Slides were incubated at $4^{\circ} \mathrm{C}$ overnight with mouse anti-IR $\beta$ monoclonal antibody (1:500), followed by secondary biotynilated goat anti-mouse IgG and streptavidin-peroxidase at $37^{\circ} \mathrm{C}$ for $30 \mathrm{~min}$ each. The reaction was revealed with 3,3'-diaminobenzidine tetrahydrochloride. Nuclei were stained with hematoxylin. For negative controls, the primary antibody was replaced with phosphate buffer saline.

\section{Oral glucose tolerance test}

At the end of the 12-week experiment, the rats were fasted overnight and fed with D-glucose by gavage at a dose of $3.0 \mathrm{~g} / \mathrm{kg}$ body weight. Blood samples were obtained by tail bleeding for determination of glucose concentrations before and at 30,60,90, and 120 min after the glucose load. The glucose concentration was determined using a Bayer Contour TS blood glucose meter with matched test strips.

Published by Bioscientifica Ltd. 


\section{Determination of serum insulin concentration}

Serum insulin concentration was determined using a competitive ELISA kit following the manufacturer's instructions. The index of homeostasis model assessment of insulin resistance (HOMA-IR) was calculated according to the following formula: (fasting serum glucose $\times$ fasting serum insulin)/22.5 (Zhao et al. 2010).

\section{Measurement of hepatic glycogen}

The content of hepatic glycogen was determined by a commercially available assay kit based on the anthrone reagent method (Jung et al. 2004). In brief, glycogen was precipitated with anhydrous ethanol and dehydrated by $98 \% \mathrm{H}_{2} \mathrm{SO}_{4}$ in order to generate furfural derivatives, which may produce a blue compound after reacting with anthrone. The optical density of the blue compound was measured at $620 \mathrm{~nm}$ using an Agilent $8453 \mathrm{UV} /$ Visible spectrophotometer (Agilent Technologies). Glycogen content of the sample was determined according to the standard curve generated by standard glucose solutions.

\section{Western blot analysis}

Tissues were homogenized in ice-cold RIPA buffer $(50 \mathrm{mM}$ Tris, pH7.4, $150 \mathrm{mMNaCl}, 1 \%$ NonidetP-40,0.1\%SDS, $1 \mathrm{mM}$ EDTA, $1 \mathrm{mM}$ PMSF, $1 \mathrm{mM} \mathrm{Na} \mathrm{VO}_{4}, 25 \mathrm{mM} \mathrm{NaF}, 10 \mu \mathrm{g} / \mathrm{mL}$ leupeptin, $10 \mu \mathrm{g} / \mathrm{mL}$ aprotinin). The supernatants were collected by centrifugation at $10,000 \mathrm{~g}$ for $10 \mathrm{~min}$ at $4^{\circ} \mathrm{C}$, and protein concentrations were determined by BCA protein assay. Equal amounts (30-50 $\mu \mathrm{g}$ ) of protein were loaded on 10\% SDS-polyacrylamide gel, separated, and transferred onto polyvinylidene difluoride membranes. The blots were probed with rabbit anti-phospho Akt $\left(S^{4}{ }^{43}\right)$ (1:1000) and rabbit anti-Akt (1:500), followed by HRP-conjugated goat anti-rabbit IgG (1:10,000). Signals were visualized by enhanced chemiluminescence detection.

\section{Immunoprecipitation}

Liver tissues were homogenized $1: 10 \mathrm{w} / \mathrm{v}$ in ice-cold lysis buffer (20 mM Tris, pH 7.5, $150 \mathrm{mM} \mathrm{NaCl}, 1 \%$ Triton X-100) supplemented with protease and phosphatase inhibitors. Homogenates were centrifuged at $10,000 \boldsymbol{g}$ for $10 \mathrm{~min}$ to remove cellular debris, and supernatants were precleared with Protein A/G plus-agarose beads. Two micrograms of rabbit anti-IR $\beta$ monoclonal antibody or rabbit anti-IRS2 antibody was incubated with $50 \mu \mathrm{L}$ Protein A/G beads overnight at $4^{\circ} \mathrm{C}$ with constant mixing. Antibody-coated beads were washed 3 times with lysis buffer to remove

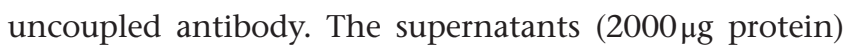
were added to the antibody-coated beads and incubated overnight at $4^{\circ} \mathrm{C}$ with constant mixing. The beads were washed with lysis buffer, resuspended in Laemmli reducing sample buffer, and pelleted by a brief centrifugation. The supernatants were loaded onto 10\% SDS-polyacrylamide gel. Phosphorylated IR $\beta$ and IRS2 were detected by Western blotting using anti-PY antibody (1:1000).

\section{Activities of hepatic enzymes}

The activities of PI3K, GSK3 $\alpha$, and GS were determined by measuring NADH oxidation in the pyruvate kinase-lactate dehydrogenase coupling system following the instruction from the manufacturer's protocols. One unit of activity is defined as the quantity of enzyme that catalyzed the oxidation of $1 \mu \mathrm{mol} \mathrm{NADH}$ to $\mathrm{NAD}^{+}$per min at $30^{\circ} \mathrm{C}$ and $\mathrm{pH} 7.5$ after incubation with different substrates, which were phosphatidylinositol 4,5-bisphosphate for PI3K, RRAAEELDSRAGSPQL for GSK3 $\alpha$, and uridine diphosphate glucose for GS, respectively. NADH was determined by measuring spectrophotometric absorbance at $340 \mathrm{~nm}$. Protein content in the enzymatic extracts was determined by the Bradford protein assay. The activity was expressed as $\mu \mathrm{mol} \mathrm{NADH} / \mathrm{min}$ per mg protein. GPa activity was determined by evaluating the reduction of $\mathrm{NADP}^{+}$to NADPH in a system containing phosphoglucomutase and glucose-6-phosphate dehydrogenase and expressed as $\mu \mathrm{mol} \mathrm{NADP} / \mathrm{min}$ per mg protein. Control experiments without substrate and with heat-denatured liver tissue extract were also performed. All of the samples were assessed in triplicate.

\section{Statistical analyses}

Data are expressed as mean \pm s.D. or median values (IQR) as appropriate. All boxplots show median values (horizontal lines inside the boxes), quartiles (box boundaries), maximum and minimum values (whiskers), and outliers (dots). Statistical Package for the Social Sciences (SPSS version 20.0) software was used for the statistical analysis. Comparison of continuous variables was performed using one-way ANOVA or the nonparametric analysis of variance Kruskal-Wallis test as appropriate. All subgroup analyses were performed using Bonferroni's correction to adjust for multiple comparisons. A $P$-value of $<0.05$ was considered to be statistically significant.

Published by Bioscientifica Ltd. 


\section{Results}

\section{Metformin alleviates liver injury in rats of NASH and cirrhosis}

At the end of the 12-week experiment, all animals in the model group developed steatohepatitis and cirrhosis, and one rat died during the experiment. Macroscopic evaluation of the livers from the control rats showed red, smooth, and shiny liver tissues, whereas the livers from the model rats displayed greasy appearances with a variety of small nodules on the surfaces. The sick appearance of the liver was largely improved by the treatment of metformin (Fig. 1A). There were no significant differences in body weight among the three groups (Fig. 1B). Animals in the model group exhibited increased liver weight and spleen weight. Metformin treatment to the cirrhosis rats reduced both the liver weight and the spleen weight (Fig. 1C).

Metformin also significantly decreased serum concentrations of Tbil, ALP, and TC, and increased
Alb level (Fig. 1D, E, F, G, H and I). For investigation of histopathological changes of the liver, H\&E and Sirius red staining were performed with representative photomicrographs shown in Fig. 2A and B. The control rats displayed the normal liver architectures. In the model group, the structure of normal hepatic lobules was severely destroyed, featured with extensive collagen deposition, fibrous septa, and cirrhotic nodules as well as with massive fatty degeneration. The lesions were significantly alleviated by metformin. A marked decrease in the severity of fat accumulation and collagen deposition was observed in the liver tissues of the metformin-treated rats.

\section{Metformin suppresses hepatic oxidative stress}

As illustrated in Fig. 2C, the level of MDA, a marker of lipid peroxidation, in the livers of the model rats was increased significantly up to 3.13-fold over control $(P<0.001)$. MDA level of the metformin-treated group
A
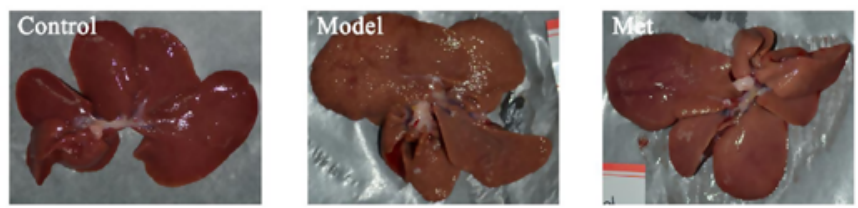

B
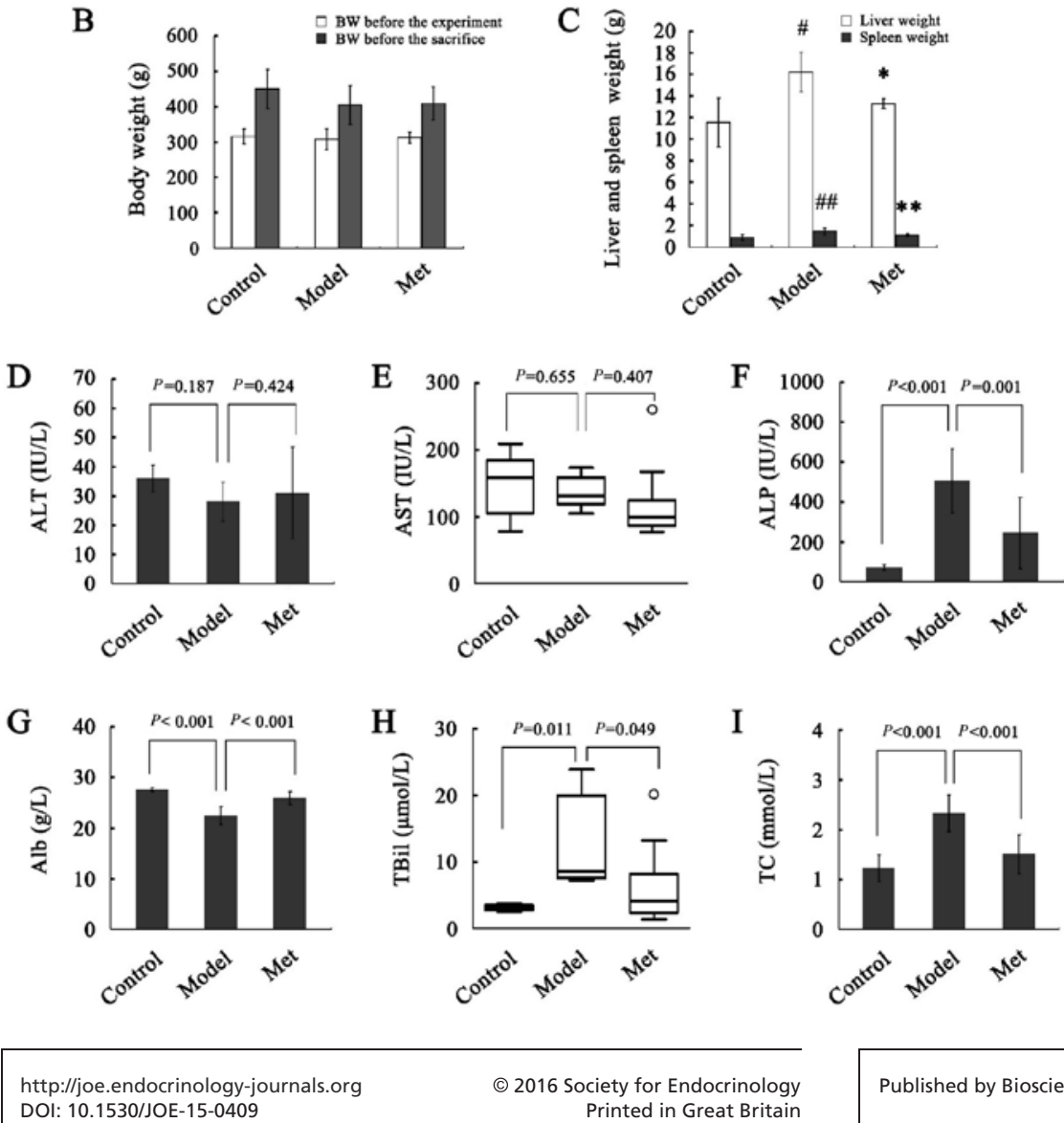

Figure 1

Metformin reduces liver weight and spleen weight and improves serum biochemical parameters of liver function. Animals and treatments are as described in Materials and Methods section. (A) Gross macroscopic appearance of the livers from the three different groups. (B) Body weights of the animals before the experiment and before being killed. (C) Liver weight and spleen weight. Liver weight, ${ }^{\sharp} P=0.003$ versus the control group, ${ }^{*} P=0.002$ versus the model group. Spleen weight, $\# P=0.007$ versus the control group, ${ }^{* *} P=0.022$ versus the model group. (D, E, F, G, H and I) Serum concentrations of ALT, AST, ALP, Alb, TBil, and TC in control rats $(n=10)$, model rats $(n=9)$ and metformin-treated rats $(n=10)$. Data are expressed as mean \pm s.D. or median values (IQR) as appropriate. A full colour version of this figure is available online at http://dx.doi.org/10.1530/ JOE-15-0409. 
A
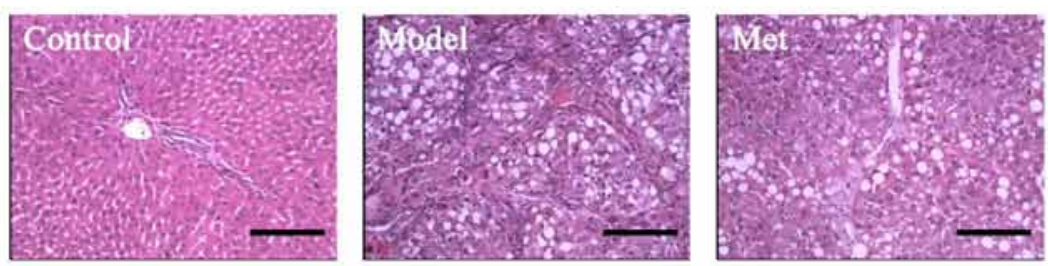

B
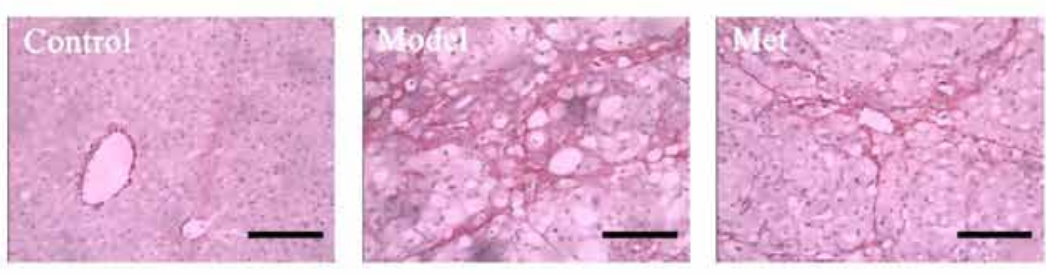

C

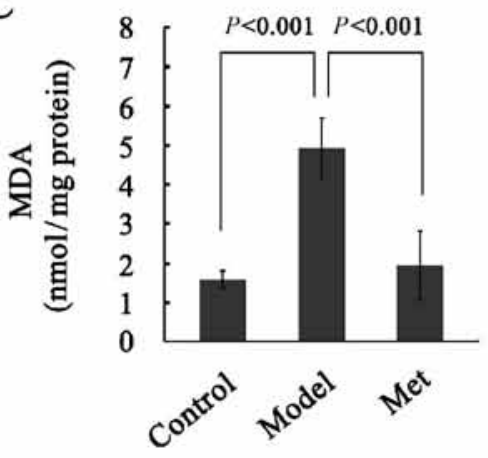

E

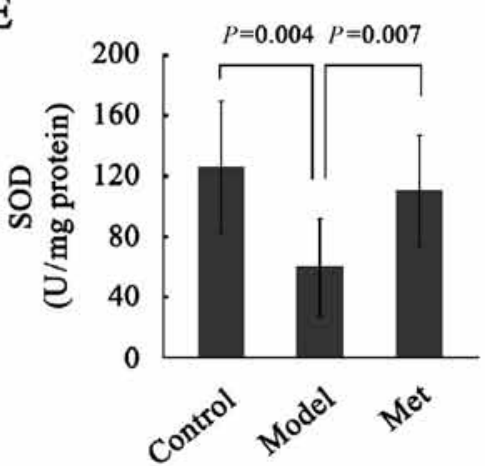

D

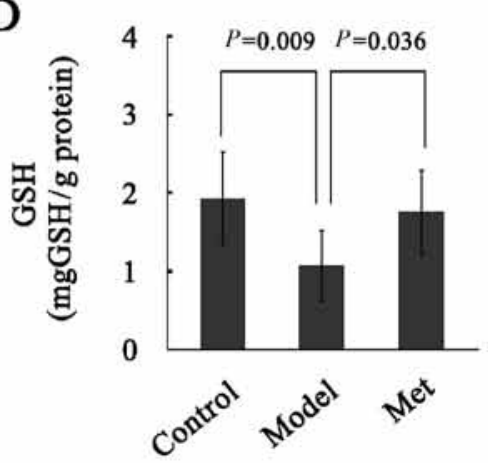

F

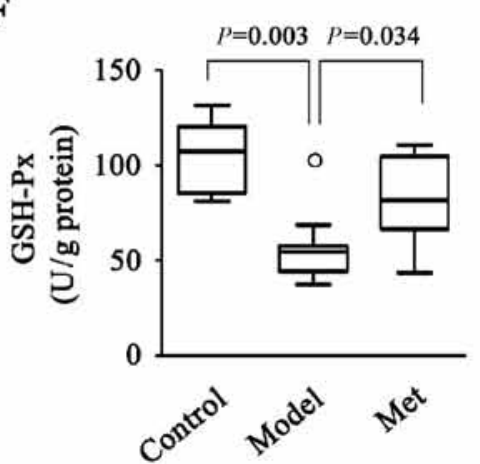

Figure 2

Metformin alleviates histopathological damages and suppresses oxidative stress in liver.

(A) Histological evaluation of liver tissues by H\&E staining. Scale bar $=100 \mu \mathrm{m}$. (B) Collagen deposition was evaluated by Sirius red staining. The red color represents the collagen fibers. Scale bar $=100 \mu \mathrm{m}$. Levels of MDA (C) and GSH (D) and activities of SOD (E) and GSH-Px (F) in the liver tissue homogenates of control rats $(n=10)$, model rats $(n=9)$, and metformin-treated rats $(n=10)$. Data are expressed as mean \pm S.D. or median values (IQR) as appropriate. A full colour version of this figure is available online at http://dx.doi. org/10.1530/JOE-15-0409. was significantly reduced by $60.73 \%$ compared with the model group $(P<0.001)$.

To evaluate the antioxidant capacity of the liver, the activities of SOD and GSH-Px and the level of GSH in the liver homogenates were measured. Compared with the control group, the activities of SOD and GSH-Px, as well as the GSH level, were significantly decreased in the model group $(P=0.004, P=0.003$, and $P=0.009$, respectively). Metformin significantly restored the activities of SOD $(P=0.007)$ and GSH-Px $(P=0.034)$ and increased the content of GSH $(P=0.036)$ (Fig. 2D, E and F).

\section{Metformin ameliorates glucose intolerance and insulin resistance in rats of NASH and cirrhosis}

OGTT was performed to evaluate the status of glucose tolerance. As illustrated in Fig. 3A, there were no significant differences in fasting serum glucose level among the three groups. After the oral glucose load, the control rats showed a rapid elevation of blood glucose, reaching a peak level of $8.9 \pm 0.2 \mathrm{mmol} / \mathrm{L}$ at $60 \mathrm{~min}$. The blood glucose levels of the cirrhosis model rats after the glucose load were significantly higher than those of the control rats and exhibited a maximal level 
A
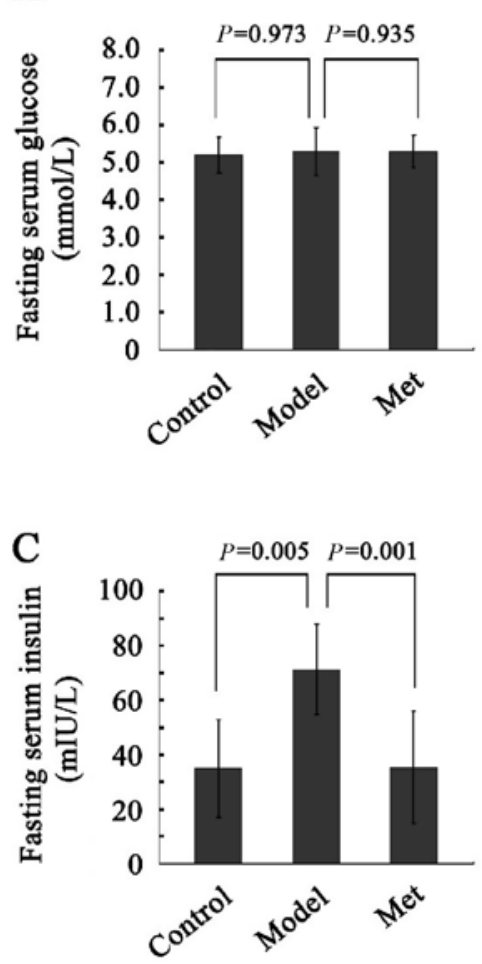

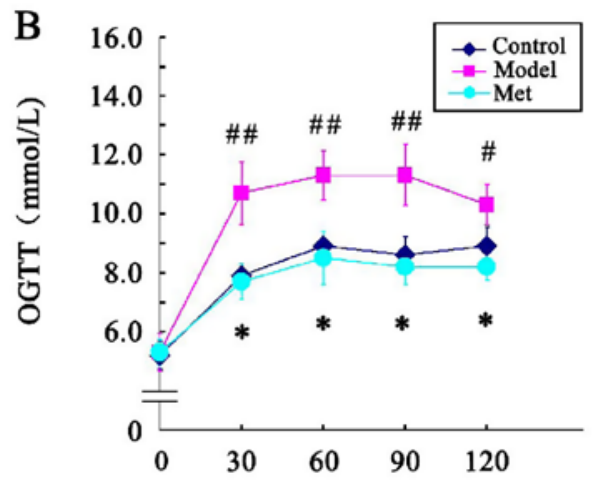

Time after oral glucose load $(\min )$

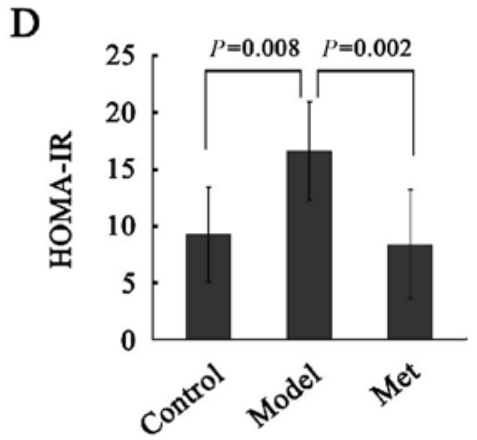

Figure 3

Metformin ameliorates glucose intolerance and insulin resistance in $\mathrm{HFHSD}$ and $\mathrm{CCl}_{4}$-induced cirrhotic rats. (A) No significant differences in fasting serum glucose levels among the three groups were detected. (B) Oral glucose tolerance test. Animals were fed with $\mathrm{D}$-glucose after overnight fasting by gavage at a dose of $3.0 \mathrm{~g} / \mathrm{kg}$ body weight. Blood glucose levels were detected prior to and at 30,60,90, and $120 \mathrm{~min}$ after the glucose load. $\# P=0.005$ versus the control group, $\# P<0.001$ versus the control group, ${ }^{*} P<0.001$ vs the model group. (C) Fasting serum insulin concentrations. (D) HOMA-IR was calculated as (fasting serum glucose $\times$ fasting serum insulin)/22.5. All data were expressed as mean \pm S.D. of 9-10 rats per group. A full colour version of this figure is available online at http:// dx.doi.org/10.1530/JOE-15-0409. of $11.3 \pm 0.8 \mathrm{mmol} / \mathrm{L}$. The impaired glucose tolerance was significantly improved by metformin treatment (Fig. 3B).

The fasting serum insulin concentration of the model rats was higher than that of the control rats
$(71.36 \pm 16.51 \mathrm{mIU} / \mathrm{L}$ vs $34.98 \pm 18.02 \mathrm{mIU} / \mathrm{L}, \quad P=0.005)$ Metformin remarkably reduced the fasting serum insulin level to $35.43 \pm 20.59 \mathrm{mIU} / \mathrm{L}(P=0.001)$ (Fig. 3C). Similarly, HOMA-IR was $9.25 \pm 4.17$ in the control, $16.59 \pm 4.37$ in the model, and $8.37 \pm 4.78$ in the metformin-treated group
A

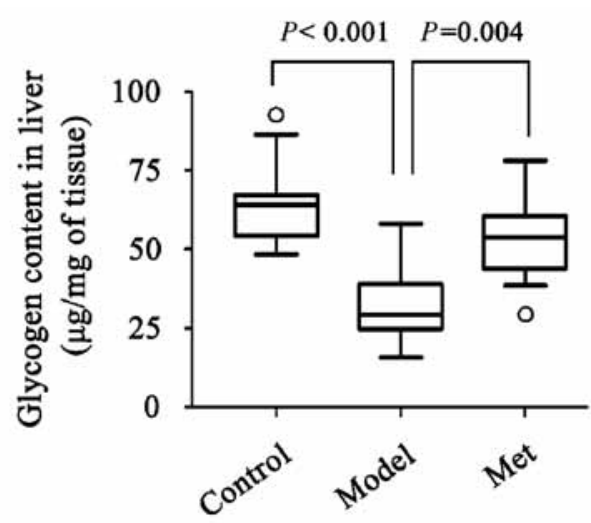

B
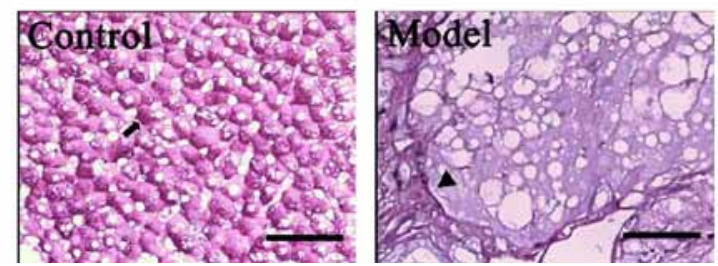

Figure 4

Metformin increases hepatic glycogen storage. (A) The glycogen in the livers of control rats $(n=10)$, model rats $(n=9)$, and metformin-treated rats $(n=10)$. The glycogen content was determined by the anthrone reagent method and was expressed as $\mu \mathrm{g} / \mathrm{mg}$ liver tissue. $P$-values are shown alongside the boxplot. (B) Representative photomicrographs show PAS staining of liver sections. The black arrow represents the glycogen particles, which were abundant in the normal control liver. The arrowhead shows fibrous septa in the livers of the model rats. In the area of severe collagen and fat accumulation, positive glycogen staining was barely observed. Scale bar $=100 \mu \mathrm{m}$. A full colour version of this figure is available online at http://dx.doi.org/10.1530/ JOE-15-0409. http://joe.endocrinology-journals.org DOI: 10.1530/JOE-15-0409
(C) 2016 Society for Endocrinology Printed in Great Britain 
(Control vs Model $P=0.008$ and Model vs Metformintreated group $P=0.002$, respectively) (Fig. 3D).

\section{Metformin increases glycogen storage in the liver}

As metformin improved glucose intolerance and insulin resistance, we next evaluated the glycogen content in the liver. As illustrated in Fig. 4A, the glycogen content of the control rats was $64.30(54.33,67.35) \mu \mathrm{g} / \mathrm{mg}$ liver tissue. Rats in the model group exhibited remarkably decreased hepatic glycogen of $29.51(24.99,39.13) \mu \mathrm{g} / \mathrm{mg}$. The glycogen of the metformin-treated rats was significantly increased to $53.93(44.02,60.54) \mu \mathrm{g} / \mathrm{mg}$.

Representative images of PAS staining were shown in Fig. 4B. The livers of the control rats displayed high PAS staining intensity, which reflected the number of glycogen particles, in the cytoplasm of hepatocytes, whereas the staining intensity in the model group was dramatically attenuated. In the area of severe collagen and fat accumulation, the positive staining was barely
A

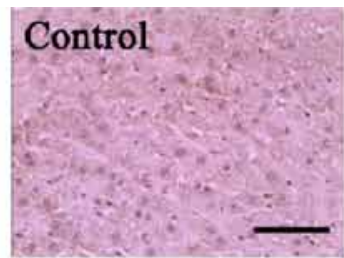

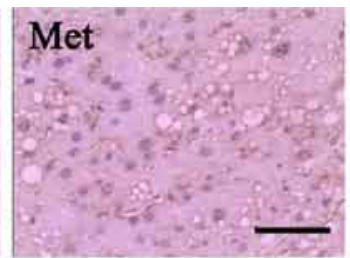

B IP: IR $\beta$ pTyr

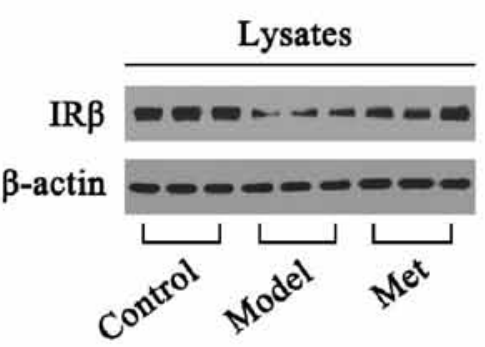

D

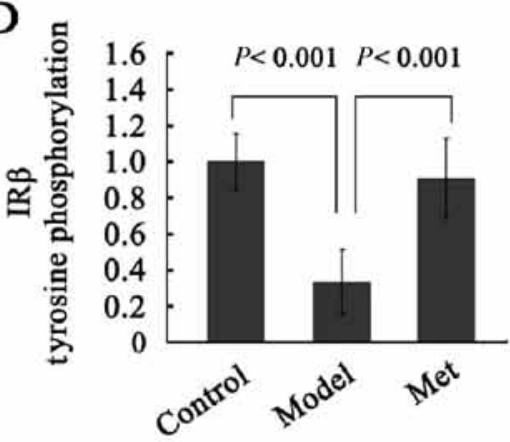

F

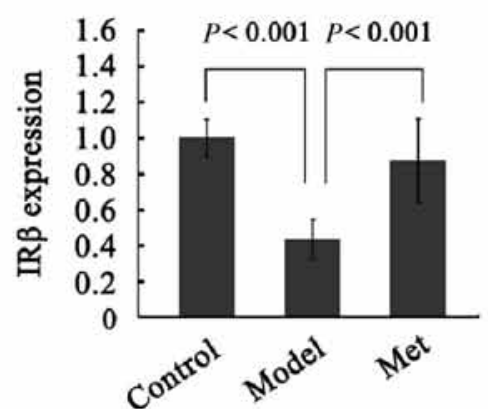

$\mathrm{C}$ pTyr

IP: IRS2

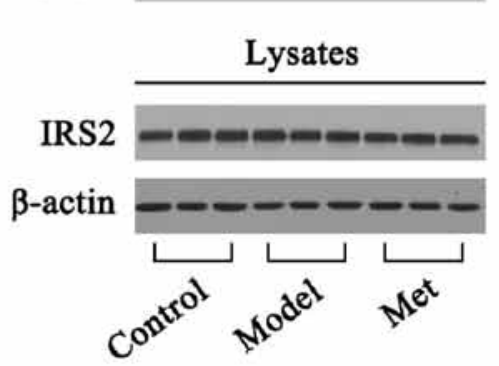

$\mathrm{E}$

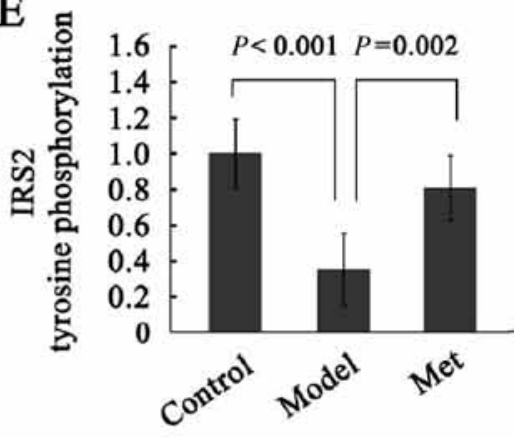

G

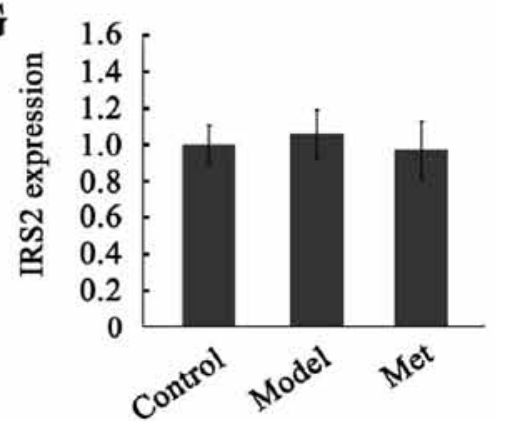

Figure 5

Metformin upregulates hepatic IR $\beta$ expression and increases tyrosine phosphorylation of IR $\beta$ and IRS2. (A) Immunohistochemical staining of IR $\beta$ in the liver. The brown color represents positive staining of IR $\beta$. Scale bar $=100 \mu \mathrm{m}$. (B and C) IR $\beta$ and IRS2 were precipitated by rabbit anti-IR $\beta$ monoclonal antibody and rabbit anti-IRS2 antibody, respectively, and immunoblotted by anti-PY antibody. IR $\beta$ and IRS 2 in the lysates were detected by rabbit anti-IR $\beta$ monoclonal antibody and rabbit anti-IRS2 antibody, respectively. $\beta$-Actin demonstrates the equal loading of proteins. ( $D$ and $E$ ) Tyrosine phosphorylations of IR $\beta$ and IRS2. The mean densities of tyrosinephosphorylated IR $\beta$ and IRS2 were normalized by the densities of total IR $\beta$ and IRS2, respectively. The control samples were assigned a value of 1 . ( $F$ and G) Graphic presentations show the expressions of IR $\beta$ and IRS2. The mean densities of IR $\beta$ and IRS 2 were normalized by that of $\beta$-actin. The control samples were assigned a value of 1 . Data are representative of at least nine rats per group. A full colour version of this figure is available online at http://dx.doi.org/10.1530/ JOE-15-0409. 
observed. The staining intensity of glycogen in the livers of the metformin-treated rats was much stronger than that in the model rats.

\section{Metformin upregulates hepatic IR $\beta$ expression}

IR consists of two extracellular $\alpha$-subunits and two transmembrane $\beta$-subunits. Figure $5 \mathrm{~A}$ shows the immunohistochemical staining of $\operatorname{IR} \beta$ in the liver. The normal livers displayed intensive brown staining of IR $\beta$ in the cytoplasm, whereas tissues from the model rats had little positive staining. Significantly increased IR $\beta$ staining in the metformin-treated group was observed compared with the model group. The results were consistent with the data of western blotting, which showed that the expression of IR $\beta$ in the livers of the model rats was $43.26 \%$ of control level
$(P<0.001)$. Metformin caused a significant increase in $\operatorname{IR} \beta$ expression up to 2.02-fold over the model group $(P<0.001)$ (Fig. 5B and F). No significant differences were detected in the expression of IRS2 among the three groups (Fig. 5C and G).

\section{Metformin increases phosphorylations of IR $\beta$, IRS2, and Akt}

By using anti-PY antibody, IR $\beta$ and IRS2 were immunoprecipitated for the detection of tyrosine phosphorylation. As shown in Fig. 5B, C, D and E, tyrosine phosphorylations of IR $\beta$ and IRS 2 in the model group were significantly decreased to 33.22 and $35.08 \%$ of control level, respectively (both $P<0.001$ ). Metformin treatment increased IR $\beta$ and IRS2 phosphorylation to 2.73-fold and 2.30-fold over the model group, respectively $(P<0.001$ and $P=0.002)$.
A

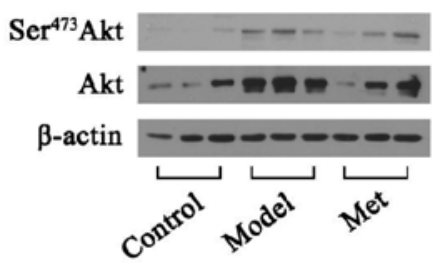

C

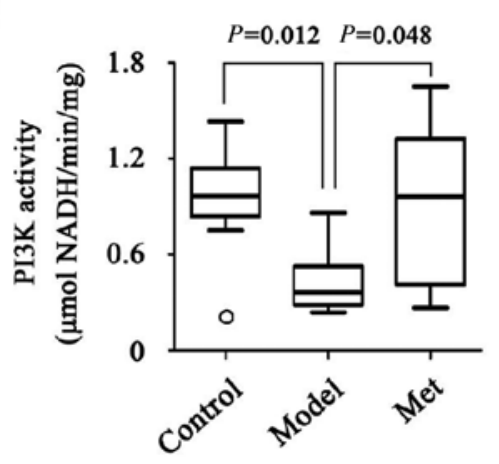

E

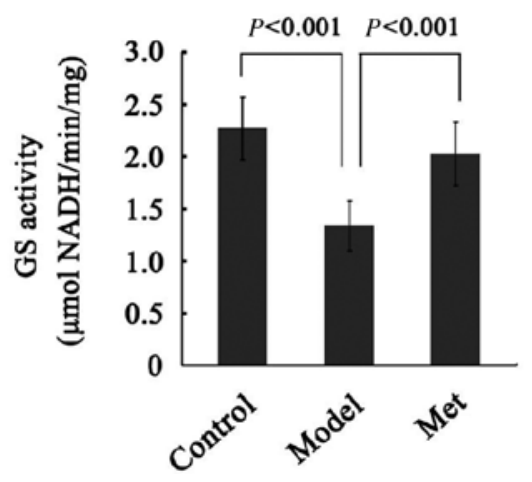

B

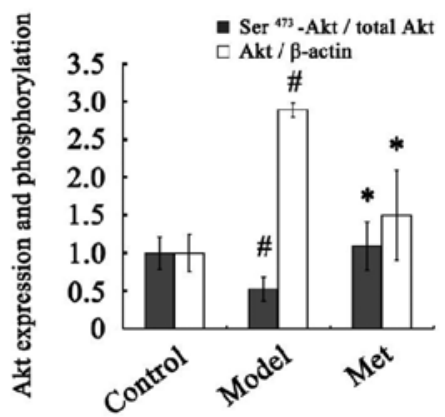

D

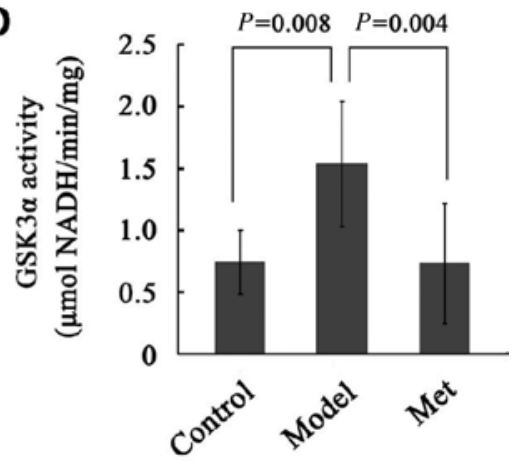

F

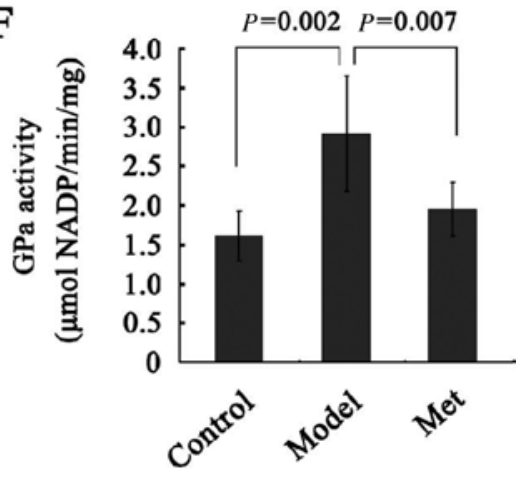

\section{Figure 6}

Metformin increases Akt phosphorylation, enhances activities of PI3K and GS, and reduces activities of GSK3 $\alpha$ and GPa in the liver. (A) Representative western blots depicting Ser ${ }^{473}$ phospho-Akt and total Akt. $\beta$-Actin demonstrates the equal loading of proteins. (B) Graphic presentations show the phosphorylation and expression of Akt. Akt phosphorylation is represented as the relative ratio of the density of Ser473-Akt against that of total Akt. Control samples were assigned a value of $1 . \# P<0.001$ vs the control, ${ }^{*} P<0.001$ vs the model group. The activities of PI3K (C), GSK3 $\alpha$ (D), GS (E), and GPa (F). All of the samples were assessed in triplicate. Data are representative of 9-10 rats per group and expressed as mean \pm S.D. or median values (IQR) as appropriate. $P$-values are shown alongside the graphs. 
As shown in Fig. 6A and B, the model rats exhibited 2.89fold higher Akt expression than the control rats. Metformin caused a considerable reduction in Akt level by $51.87 \%$. By contrast, Ser ${ }^{473}$ phosphorylation of Akt in the model rats was significantly decreased to $52.21 \%$ of control level. In the metformin-treated group, Ser ${ }^{473}$-Akt was significantly increased and was 2.09-fold over the model group.

\section{Metformin causes an increase in activities of PI3K and GS, whereas it causes a decrease in activities of GSK3 $\alpha$ and GPa}

As illustrated in Fig. 6C, D, E and F, significantly decreased activities of PI3K and GS were detected in the liver tissues of the model rats $(P=0.012$ and $P<0.001$, respectively). In the metformin-treated group, the activities of PI3K and GS were higher than that of the model group ( $P=0.048$ and $P<0.001$, respectively). By contrast, the activities of GSK3 $\alpha$ and GPa were significantly increased up to 2.07-fold and 1.81-fold over control $(P=0.008$ and $P=0.002)$, respectively. Metformin treatment caused a $52.3 \%$ reduction in GSK3 $\alpha$ activity $(P=0.004)$ and $33.2 \%$ reduction in GPa activity $(P=0.007)$.

\section{Discussion}

To date, the influence of metformin on hepatic glycogen synthesis has been still controversial (Radziuk et al. 2003). By developing an insulin-resistant rat model of NASH and cirrhosis, this study has provided evidence that metformin increases hepatic glycogen storage. This action of metformin is attributed to the increased expression and phosphorylation of IR and the augmentation of downstream IRS2/PI3K/Akt signaling transduction pathway. To the best of our knowledge, this is for the first time indicating that metformin upregulates hepatic IR expression and the downstream IRS2/PI3K/Akt pathway in vivo.

Animal models are essential research tools for investigating the pathophysiology of hepatic insulin resistance. Dietary-induced NAFLD rodent models, which vary in diet compositions, duration of feeding, animal strain, sex, and age, are the most widely used according to the previous studies (Kucera \& Cervinkova 2014). A variety of genetic models of NAFLD are more expensive and less available (Qi et al. 2005, Nagarajan et al. 2012). These models failed to show high reproducibility with regard to the development of insulin resistance, and few of them showed the progress from NASH to fibrosis even cirrhosis (Larter \& Yeh 2008). During our preliminary experiments, rats fed with HFHSD for 12 weeks failed to acquire insulin resistance based on HOMA-IR assessment, whereas increased HOMA-IR was detected in those fed with HFHSD for 24 weeks. As 24-week feeding is time consuming, we attempt to create a new insulin-resistant rat model of liver disease in a shorter period of time. $\mathrm{CCl}_{4}$ is associated with increased lipid peroxidation, impaired hepatic antioxidant activity, and necrosis of hepatocytes, because it is metabolized into trichloromethyl radical (Weber et al. 2003). Available study indicated that hepatic glycogen content was considerably reduced in the rat model of cirrhosis induced by intraperitoneal injection of $\mathrm{CCl}_{4}$ for 19 weeks. However, increased early insulin secretion might compensate adequately for hepatic insulin resistance (Arai et al. 2010). $\mathrm{CCl}_{4}$ usually caused a dramatic reduction in body weight and food intake. Thus, we designed a rat model, not only presenting steatohepatitis and cirrhosis but also exhibiting insulin resistance, by HFHSD feeding in combination with subcutaneous injection of $\mathrm{CCl}_{4}$ for 12 weeks. More importantly, the remarkable weight loss and low food intake that happened in cirrhosis rats exposed to $\mathrm{CCl}_{4}$ alone were not observed. The histological observations were accompanied by increased serum TC level and dropped albumin level, whereas there was no change in ALT and AST levels, indicating that liver function was severe deteriorated. We suggest that the successful development of insulin resistance may depend on the severity of liver function injury and histopathological damage. The rats fed by HFHSD in combination with $\mathrm{CCl}_{4}$ exposure appear more susceptible to the development of insulin resistance.

Our model rats were treated with metformin at a clinically relevant concentration, and marked beneficial effects were observed. Apparently, the macroscopic appearance of steatotic and cirrhotic liver was considerably improved. Liver weight and spleen weight were decreased. Liver histological damages were significantly alleviated with serum level of TC decreased and Alb level increased. Glucose intolerance and insulin resistance were effectively improved. Moreover, metformin treatment significantly increased hepatic glycogen storage. To date, the preponderance of the literature has suggested that the major therapeutic action of metformin in the liver is on suppressing gluconeogenesis, whereas its influence on glycogenesis is likely quite small (Radziuk et al. 2003). Available data also demonstrated that metformin impaired glycogen synthesis in isolated rat hepatocytes (Otto et al. 2003). However, increased hepatic glycogen content in response to metformin was detected in high-fat-fed insulin-resistant rat model and in hepatoma cells (Purrello et al. 1988, Mithieux et al. 2002). These

Published by Bioscientifica Ltd 
conflicting results may be attributable to the differences in research models and methodology.

Limited studies have evaluated the effect of metformin on hepatic insulin signaling transduction pathway. In chronic insulin-exposed HepG2 cells, the reduction in phosphorylation of IR $\beta$ and IRS 2 was reversed by metformin (Yuan et al. 2003). Gunton and coworkers suggested that metformin caused an increase in tyrosine phosphorylation of the $\beta$-subunit in whole IR partially purified from primary human hepatocytes as well as in Huh7 human hepatoma cells (Gunton et al. 2003). These data were all based on in vitro studies. In our insulin-resistant rat model of NASH and cirrhosis, subdued IRS2/PI3K/Akt signaling pathway in the liver was detected. The expression of IR $\beta$; phosphorylations of IR $\beta$, IRS2, and Akt; and activities of PI3K and GS were significantly reduced, whereas the activities of GSK3 $\alpha$ and GPa were increased. These molecular events were significantly reversed by metformin treatment. The most important novel finding in this study was that metformin upregulates IR expression in the steatotic and cirrhotic liver. Although increased phosphorylation of $\operatorname{IR} \beta$ by metformin in vitro has been reported, no literature can be found showing that metformin directly affects the numbers of IR in the liver. We consider the increased activation of IRS2/PI3K/Akt signaling pathway as a consequence of the increased hepatic IR expression.

To date, considerable clinical studies have investigated the efficacy of metformin on NAFLD. It aids weight loss, improves biochemical and metabolic parameters, and reduces insulin resistance in patients with NAFLD (Haukeland et al. 2009, Zheng et al. 2015). The findings of Zhang and coworkers indicated that continuation of metformin improves the survival of NASH-related cirrhotic patients with diabetes by reducing the risk of death by $57 \%$ (Zhang et al. 2014). However, the majority of clinical evidence denied the effectiveness of this drug in ameliorating liver histological damages. Thus, metformin is not recommended as a specific treatment for NAFLD in clinical practice guidelines (Chalasani et al. 2012). By contrast, the data based on animal studies have shown that metformin improves hepatic steatosis, liver inflammation, and to a certain degree fibrosis (Lin et al. 2000, Woo et al. 2014, Tripathi et al. 2015). These results are consistent with the data of this study. In our model rats of NASH and cirrhosis, the liver damages, including steatosis and collagen deposition, were significantly improved by metformin. Interestingly, available evidence indicated that metformin alleviated liver fibrosis in $\mathrm{CCl}_{4}$ cirrhotic rats, but not in cirrhotic rats induced by common bile duct ligation (Tripathi et al. 2015). Published data also suggested that metformin prevented hepatic steatosis by regulating the expression of adipose differentiation-related protein (Liu et al. 2014). AMPK, which can be activated by metformin, is originally discovered by its ability to inhibit fatty acid and cholesterol synthesis (Carlson \& Kim 1973). This study indicated that metformin suppressed the oxidative stress and restored the antioxidant capacity of the liver, which may result in improved hepatic lipid metabolism and decreased steatosis. Thus, it is reasonable for us to speculate that metformin may slow the progression of fibrosis by attenuating steatohepatitis. The improvement of liver histological alterations by metformin potentially leads to the upregulation of hepatic IR expression.

In summary, this study supports the theory that metformin improves lipid peroxidation and histological damages of the liver, alleviates glucose intolerance and insulin resistance, and increases hepatic glycogen storage. Importantly, we present novel evidence that metformin increases IR expression in the liver, resulting in the enhancement of the downstream IRS2/PI3K/Akt signaling pathway. The data are derived from in vivo studies based on animal models and enriches the existing literatures on mechanisms by which metformin improves hepatic glucose metabolism.

\section{Declaration of interest}

The authors declare that there is no conflict of interest that could be perceived as prejudicing the impartiality of the research reported.

\section{Funding}

This work was supported by the National Natural Science Foundation of China (81102707) and Medical Science and Technology Project of Zhejiang Province (2013KYA165).

\section{Acknowledgment}

The authors thank Professor Lv Hua for his assistance in statistical analysis.

\section{References}

Agius L 2015 Role of glycogen phosphorylase in liver glycogen metabolism. Molecular Aspects of Medicine 46 34-45. (doi:10.1016/j. mam.2015.09.002)

Ahmadieh H \& Azar ST 2014 Liver disease and diabetes: association, pathophysiology, and management. Diabetes Research and Clinical Practice 104 53-62. (doi:10.1016/j.diabres.2014.01.003)

Arai H, Awane N, Mizuno A, Fukaya M, Sakuma M, Harada N, Kawaura A, Yamamoto H, Okumura H, Taketani Y, et al. 2010 Increasing early insulin secretion compensate adequately for hepatic insulin resistance in CCl4-induced cirrhosis rats. Journal of Medical Investigation 57 54-61. (doi:10.2152/jmi.57.54)

Published by Bioscientifica Ltd. 
Beurel E, Grieco SF \& Jope RS 2015 Glycogen synthase kinase-3 (GSK3): regulation, actions, and diseases. Pharmacology \& Therapeutics 148 114-131. (doi:10.1016/j.pharmthera.2014.11.016)

Cao J, Meng S, Chang E, Beckwith-Fickas K, Xiong L, Cole RN, Radovick S, Wondisford FE \& He L 2014 Low concentrations of metformin suppress glucose production in hepatocytes through AMPactivated protein kinase (AMPK). Journal of Biological Chemistry 289 20435-20446. (doi:10.1074/jbc.M114.567271)

Carlson CA \& Kim KH 1973 Regulation of hepatic acetyl coenzyme A carboxylase by phosphorylation and dephosphorylation. Journal of Biological Chemistry 248 378-380.

Chalasani N, Younossi Z, Lavine JE, Diehl AM, Brunt EM, Cusi K, Charlton M \& Sanyal AJ 2012 The diagnosis and management of non-alcoholic fatty liver disease: practice Guideline by the American Association for the Study of Liver Diseases, American College of Gastroenterology, and the American Gastroenterological Association. Hepatology 55 2005-2023. (doi:10.1002/hep.25762)

Dyson J \& Day C 2014 Treatment of non-alcoholic fatty liver disease. Digestive Diseases 32 597-604. (doi:10.1159/000360511)

Gunton JE, Delhanty PJ, Takahashi S \& Baxter RC 2003 Metformin rapidly increases insulin receptor activation in human liver and signals preferentially through insulin-receptor substrate-2. Journal of Clinical Endocrinology \& Metabolism 88 1323-1332.

Haukeland JW, Konopski Z, Eggesbø HB, von Volkmann HL, Raschpichler G, Bjøro K, Haaland T, Løberg EM \& Birkeland K 2009 Metformin in patients with non-alcoholic fatty liver disease: a randomized, controlled trial. Scandinavian Journal of Gastroenterology 44 853-860. (doi:10.1080/00365520902845268)

He L, Sabet A, Djedjos S, Miller R, Sun X, Hussain MA, Radovick S \& Wondisford FE 2009 Metformin and insulin suppress hepatic gluconeogenesis through phosphorylation of CREB binding protein. Cell 137 635-646. (doi:10.1016/j.cell.2009.03.016)

Ide T, Shimano H, Yahagi N, Matsuzaka T, Nakakuki M, Yamamoto T, Nakagawa Y, Takahashi A, Suzuki H, Sone H, et al. 2004 SREBPs suppress IRS-2-mediated insulin signalling in the liver. Nature Cell Biology 6 351-357. (doi:10.1038/ncb1111)

Jung UJ, Lee MK, Jeong KS \& Choi MS 2004 The hypoglycemic effects of hesperidin and naringin are partly mediated by hepatic glucoseregulating enzymesin C57BL/KsJ-db/db mice. Journal of Nutrition 134 2499-2503.

Kucera O \& Cervinkova Z 2014 Experimental models of non-alcoholic fatty liver disease in rats. World Journal of Gastroenterology 20 8364-8376. (doi:10.3748/wig.v20.i26.8364)

Larter CZ \& Yeh MM 2008 Animal models of NASH: getting both pathology and metabolic context right. Journal of Gastroenterology and Hepatology 23 1635-1648. (doi:10.1111/j.1440-1746.2008.05543.x)

Lin HZ, Yang SQ, Chuckaree C, Kuhajda F, Ronnet G \& Diehl AM 2000 Metformin reverses fatty liver disease in obese, leptin-deficient mice. Nature Medicine 6 998-1003. (doi:10.1038/79697)

Liu F, Wang C, Zhang L, Xu Y, Jang L, Gu Y, Cao X, Zhao X, Ye J \& Li Q 2014 Metformin prevents hepatic steatosis by regulating the expression of adipose differentiation-related protein. International Journal of Molecular Medicine 33 51-58. (doi:10.3892/ijmm.2013.1560)

Madiraju AK, Erion DM, Rahimi Y, Zhang XM, Braddock DT, Albright RA, Prigaro BJ, Wood JL, Bhanot S, MacDonald MJ, et al. 2014 Metformin suppresses gluconeogenesis by inhibiting mitochondrial glycerophosphate dehydrogenase. Nature 510 542-546. (doi:10.1038/ nature13270)

Mithieux G, Guignot L, Bordet JC \& Wiernsperger N 2002 Intrahepatic mechanisms underlying the effect of metformin in decreasing basal glucose production in rats fed a high-fat diet. Diabetes 51 139-143. (doi:10.2337/diabetes.51.1.139)
Nagarajan P, Mahesh Kumar MJ, Venkatesan R, Majundar SS \& Juyal RC 2012 Genetically modified mouse models for the study of nonalcoholic fatty liver disease. World Journal of Gastroenterology $\mathbf{1 8}$ 1141-1153. (doi:10.3748/wjg.v18.i11.1141)

Otto M, Breinholt J \& Westergaard N 2003 Metformin inhibits glycogen synthesis and gluconeogenesis in cultured rat hepatocytes. Diabetes, Obesity and Metabolism 5 189-194. (doi:10.1046/j.14631326.2003.00263.x)

Pessin JE \& Saltiel AR 2000 Signaling pathways in insulin action: molecular targets of insulin resistance. Journal of Clinical Investigation 106 165-169.

Purrello F, Gullo D, Buscema M, Pezzino V, Vigneri R \& Goldfine ID 1988 Metformin enhances certain insulin actions in cultured rat hepatoma cells. Diabetologia 31 385-389. (doi:10.1007/BF02341508)

Qi NR, Wang J, Zidek V, Landa V, Mlejnek P, Kazdová L, Pravenec M \& Kurtz TW 2005 A new transgenic rat model of hepatic steatosis and the metabolic syndrome. Hypertension 45 1004-1011. (doi:10.1161/01. HYP.0000161995.64192.2b)

Radziuk J \& Pye S 2001 Hepatic glucose uptake, gluconeogenesis and the regulation of glycogen synthesis. Diabetes/Metabolism Research and Reviews 17 250-272. (doi:10.1002/dmrr.217)

Radziuk J, Bailey CJ, Wiernsperger NF \& Yudkin JS 2003 Metformin and its liver targets in the treatment of type 2 diabetes. Current Drug Targets: Immune, Endocrine \& Metabolic Disorders 3 151-169.

Saitoh Y, Terada N, Saitoh S, Ohno N, Fujii Y \& Ohno S 2010 Histochemical approach of cryobiopsy for glycogen distribution in living mouse livers under fasting and local circulation loss conditions. Histochemistry and Cell Biology 133 229-239. (doi:10.1007/s00418-009-0663-4)

Tripathi DM, Erice E, Lafoz E, Garcia-Caldero H, Sarin SK, Bosch J, Gracia-Sancho J \& Garcia-Pagan JC 2015 Metformin reduces hepatic resistance and portal pressure in cirrhotic rats. American Journal of Physiology: Gastrointestinal and Liver Physiology 309 G301-G309. (doi:10.1152/ajpgi.00010.2015)

Valverde AM, Burks DJ, Fabregat I, Fisher TL, Carretero J, White MF \& Benito M 2003 Molecular mechanisms of insulin resistance in IRS-2-deficient hepatocytes. Diabetes 52 2239-2248. (doi:10.2337/ diabetes.52.9.2239)

Viollet B, Guigas B, Sanz Garcia N, Leclerc J, Foretz M \& Andreelli F 2012 Cellular and molecular mechanisms of metformin: an overview. Clinical Science 122 253-270. (doi:10.1042/CS20110386)

Weber LW, Boll M \& Stampfl A 2003 Hepatotoxicity and mechanism of action of haloalkanes: carbon tetrachloride as a toxicological model. Critical Reviews in Toxicology 33 105-136. (doi:10.1080/713611034)

Woo SL, Xu H, Li H, Zhao Y, Hu X, Zhao J, Guo X, Guo T, Botchlett R, Qi T, et al. 2014 Metformin ameliorates hepatic steatosis and inflammation without altering adipose phenotype in dietinducedobesity. PLOS ONE 9 e91111. (doi:10.1371/journal. pone.0091111)

Yuan L, Ziegler R \& Hamann A 2003 Metformin modulates insulin postreceptor signaling transduction in chronically insulin-treated Hep G2 cells. Acta Pharmaceutica Sinica 24 55-60.

Zhang X, Harmsen WS, Mettler TA, Kim WR, Roberts RO, Therneau TM, Roberts LR \& Chaiteerakij R 2014 Continuation of metformin use after a diagnosis of cirrhosis significantly improves survival of patients with diabetes. Hepatology 60 2008-2016. (doi:10.1002/hep.27199)

Zhao J, Zhou G, Li M, Li W, Lü J, Xiong L, Liang L, Zhao Y, Xu D \& Yu J 2010 A novel non-alcoholic steatohepatitis animal model featured with insulin resistance, hepatic inflammation and fibrosis. Scandinavian Journal of Gastroenterology 45 1360-1371. (doi:10.3109/0 0365521.2010.497938)

Zheng J, Woo SL, Hu X, Botchlett R, Chen L, Huo Y \& Wu C 2015 Metformin and metabolic diseases: a focus on hepatic aspects. Frontiers in Medicine 9 173-186. (doi:10.1007/s11684-015-0384-0)

Received in final form 5 February 2016

Accepted 3 March 2016

Accepted Preprint published online 3 March 2016 http://joe.endocrinology-journals.org

DOI: 10.1530/JOE-15-0409
C 2016 Society for Endocrinology Printed in Great Britain 